\title{
Eruptive Nevi Mimicking Wart-Like Lesions under Selective BRAF Inhibition in a 37-Year-Old Female Melanoma Patient
}

\author{
Laurenz Schmitt $^{\mathrm{a}} \quad$ Tamara Schumann $^{\mathrm{a}}$ Oliver Inhoff $^{\mathrm{a}}$ Christoph Löser $^{\mathrm{a}}$ \\ Martin Rebel $^{b} \quad$ Edgar Dippel $^{\mathrm{a}}$ \\ Departments of ${ }^{a}$ Dermatology and ${ }^{b}$ Pathology, Klinikum Ludwigshafen, \\ Ludwigshafen am Rhein, Germany
}

\section{Key Words}

Vemurafenib - BRAF inhibitor - Eruptive nevi - Side effects - Melanoma in situ - Zelboraf .

Melanoma $\cdot$ Wart-like lesions

\begin{abstract}
Background: The BRAF inhibitor vemurafenib is state of the art in therapy of patients with malignant melanoma in non-resectable stage III or stage IV and evidence of oncogenetic BRAF mutation. Multiple cutaneous side effects like rash and keratoacanthoma-like lesions have been described so far. Case Report: We report a patient who presented multiple wartlike lesions under therapy with vemurafenib. Histologically we have seen multiple melanocytic nevi with a wart-like appearance. One melanoma in situ developed on the left forearm. Conclusions: Eruptive nevi and induction of melanoma may be a further side effect in patients undergoing a therapy with BRAF inhibitors.
\end{abstract}

\section{Introduction}

Advanced melanoma has a poor prognosis due to its resistance to traditional chemotherapeutics, leading to the search for alternative treatment approaches. With the finding that approximately $50 \%$ of melanomas harbor an activating mutation in the serine/threonine-protein kinase BRAF gene, inhibition of mutated BRAF represented an attractive and innovative focus for the development of novel targeted therapy potentially benefiting a large proportion of melanoma patients. Vemurafenib, a novel selective small 
molecule inhibitor of BRAF, has recently been shown to be effective in the treatment of melanomas harboring the BRAF V600E mutation. Successful treatment of metastatic melanoma with vemurafenib is not without significant adverse effects. Selective BRAF inhibitor therapy is associated with the development of malignant and benign growths, including keratoacanthoma-like squamous cell carcinomas, warty dyskeratomas, and verrucous keratoses, along with widespread eruptions and histologic features of acantholytic dyskeratosis [1]. Photosensitivity sometimes resulting in blistering reactions has been documented [2]. Palmar-plantar dysesthesia occurred in several patients [3]. Keratosis pilaris-like eruptions occur in roughly one third of patients treated [4]. We report a case of a female patient with metastatic melanoma treated with vemurafenib, who developed multiple melanocytic nevi with clinical 'wart-like' aspect and one melanoma in situ.

\section{Case Report}

A 37-year-old female patient affected by BRAF E600-positive metastatic melanoma under treatment with vemurafenib for a few days developed multiple melanocytic nevi with a clinical 'wart-like' aspect (fig. 1, fig. 2). The development of the multiple skin lesions appeared at day 20 after initiation of treatment with vemurafenib. In addition, a rash (grade III), arthralgia, erythema nodosum-like lesions at the thigh and a melanoma in situ on the left forearm have been observed. The melanoma in situ has been BRAF wild-type. After tangential excisions of the nevi, excision of the secondary melanoma in situ and one week discontinuation of therapy, the treatment with vemurafenib was restarted in a reduced dose of $720 \mathrm{mg}$ instead of $960 \mathrm{mg}$ twice daily. Since the follow-up period of 40 weeks no further adverse events have been noted under this treatment so far. Rash, erythema nodosum-like lesions on the tight and arthralgia declined under the therapy with topical ointment of corticosteroids and systemic NSAR.

\section{Discussion}

Mutant BRAF led to deregulated activation of downstream MEK/ERK effectors in melanoma patients. Recently published papers illustrated that selective inhibition of mutant BRAF with vemurafenib induced rapid clinical response and prolonged progression-free and overall survival in patients with BRAF V600E mutation-positive advanced melanoma [4]. However, it has been shown that adverse events of BRAF inhibitors such as the development of cutaneous squamous cell carcinoma and keratoacanthomas suggest the possibility that activating pathways could also be induced [1]. Zimmer et al. [5] lately observed 12 newly diagnosed melanomas and 10 dysplastic nevi in 11 patients under selective BRAF inhibition.

BRAF serine/threonine kinase is a member of the RAF kinase family involved in the RAS/RAF/MEK/ERK kinase cascade, which regulates cellular differentiation and proliferation [6]. BRAF protein kinase mutations are associated with a wide range of malignancies, including up to 70 percent of melanomas, 40 to 70 percent of papillary or anaplastic thyroid cancers and smaller percentages of various other cancers $[3,7]$. One specific missense mutation, valine to glutamic acid single substitution at position 600 , constitutes 80 to 90 percent of reported BRAF mutations and leads to hyperactivation of the cascade $[4,6]$. The identification of the importance of BRAF has led to the development of many new anticancer drugs. Vemurafenib is one such drug that specifically inhibits the BRAF V600E mutation. The phase 1 trial of this drug showed complete or partial tumor regression in 81 percent of 
Schmitt et al.: Eruptive Nevi Mimicking Wart-Like Lesions under Selective BRAF Inhibition in a 37-Year-Old Female Melanoma Patient

patients with the BRAF V600E mutation, whereas the phase 3 trial showed a relative reduction in risk of death of 63 percent and a relative reduction in risk of tumor progression of 74 percent as compared to dacarbazine $[3,4]$. The administration of this drug is accompanied by many adverse effects, the most common of which are arthralgia (21\%), rash (18\%), and fatigue $(13 \%)$. Cutaneous effects are also common, including pruritus, alopecia, hyperkeratosis, keratoacanthoma and squamous cell carcinoma [4].

These cutaneous effects are also associated with use of sorafenib, a nonspecific RAF kinase inhibitor that has been studied in the treatment of melanoma and is widely used for the treatment of hepatocellular carcinoma and renal cell carcinoma [8]. A study of the cutaneous effects of sorafenib by Arnault et al. [9] described the lesions as a continuum from benign follicular cystic lesions to keratoacanthomas to malignant squamous cell carcinomas. The same study showed that in cells that possess the non-mutated wild-type BRAF protein, BRAF inhibitors cause a paradoxical increase in ERK phosphorylation, leading to increased MAP kinase pathway activity. It has been hypothesized that this increase in signaling is caused by the dimerization of RAF proteins leading to activation of CRAF in combination with a second activation signal [10]. This dimerization coupled with preexisting mutations as well as additional damaging changes give rise to the range of cutaneous effects seen. Multiple nevi with wart-like appearance have not been reported for patients treated with vemurafenib yet. Until now, there has been one case reporting on eruptive nevi and keratosis pilaris-like lesions [11]. Patients undergoing treatment with selective BRAF inhibition are at risk to develop secondary malignant melanoma [5]. It is therefore recommended that these patients are very closely monitored to control any adverse events that may be caused.

\section{Disclosure Statement}

The authors declare no conflict of interest.

\section{References}

1 Oberholzer PA, Kee D, Dziunycz P, et al: RAS mutations are associated with the development of cutaneous squamous cell tumors in patients treated with RAF inhibitors. J Clin Oncol 2012;30:316-321.

2 Dummer R, Rinderknecht J, Goldinger SM: Ultraviolet A and photosensitivity during vemurafenib therapy. N Engl J Med 2012;366:480-481.

-3 Flaherty KT, Puzanov I, Kim KB, Ribas A, McArthur GA, Sosman JA, et al: Inhibition of mutated, activated BRAF in metastatic melanoma. N Engl J Med 2010;363:809-819.

-4 Chapman PB, Hauschild A, Robert C, et al: Improved survival with vemurafenib in melanoma with BRAF V600E mutation. N Eng J Med 2011;364:2507-2516.

5 Zimmer L, Hillen U, Livingstone E, et al: Atypical melanocytic proliferations and new primary melanomas in patients with advanced melanoma undergoing selective BRAF inhibition. J Clin Oncol 2012;30:2375-2383.

6 Wellbrock C, Hurlstone A: BRAF as therapeutic target in melanoma. Biochem Pharmacol 2010;80:561-567.

7 Haenssle HA, Kraus SL, Brehmer F, Kretschmer L, Völker B, Asper H, Kapp A, Gutzmer R: Dynamic changes in nevi of a patient with melanoma treated with vemurafenib: importance of sequential dermoscopy. Arch Dermatol 2012;148:1183-1185.

8 Bennani-Lahlou M, Mateus C, Escudier B, et al: Eruptive nevi associated with sorafenib treatment. Ann Dermatol Venereol 2008;135:672-674.

-9 Arnault JP, Mateus C, Escudier B, et al: Skin tumors induced by sorafenib; paradoxical RAS-RAF pathway activation and oncogenic mutations of HRAS, TP53, and TGFBR1. Clin Cancer Res 2012;18:263-272.

10 Poulikakos PI, Zhang C, Bollag G, Shokat KM, Rosen N: RAF inhibitors transactivate RAF dimers and ERK signalling in cells with wild-type BRAF. Nature 2010;464:427-430.

11 Ma L, Dominguez AR, Collins GR, Kia KF, Cockerell CJ: Hidradenitis Suppurativa, Eruptive Melanocytic Nevi, and Keratosis Pilaris-like Eruption in a Patient Treated With Vemurafenib. Arch Dermatol 2012;148:14281429. 


\section{Case Reports in Dermatology}
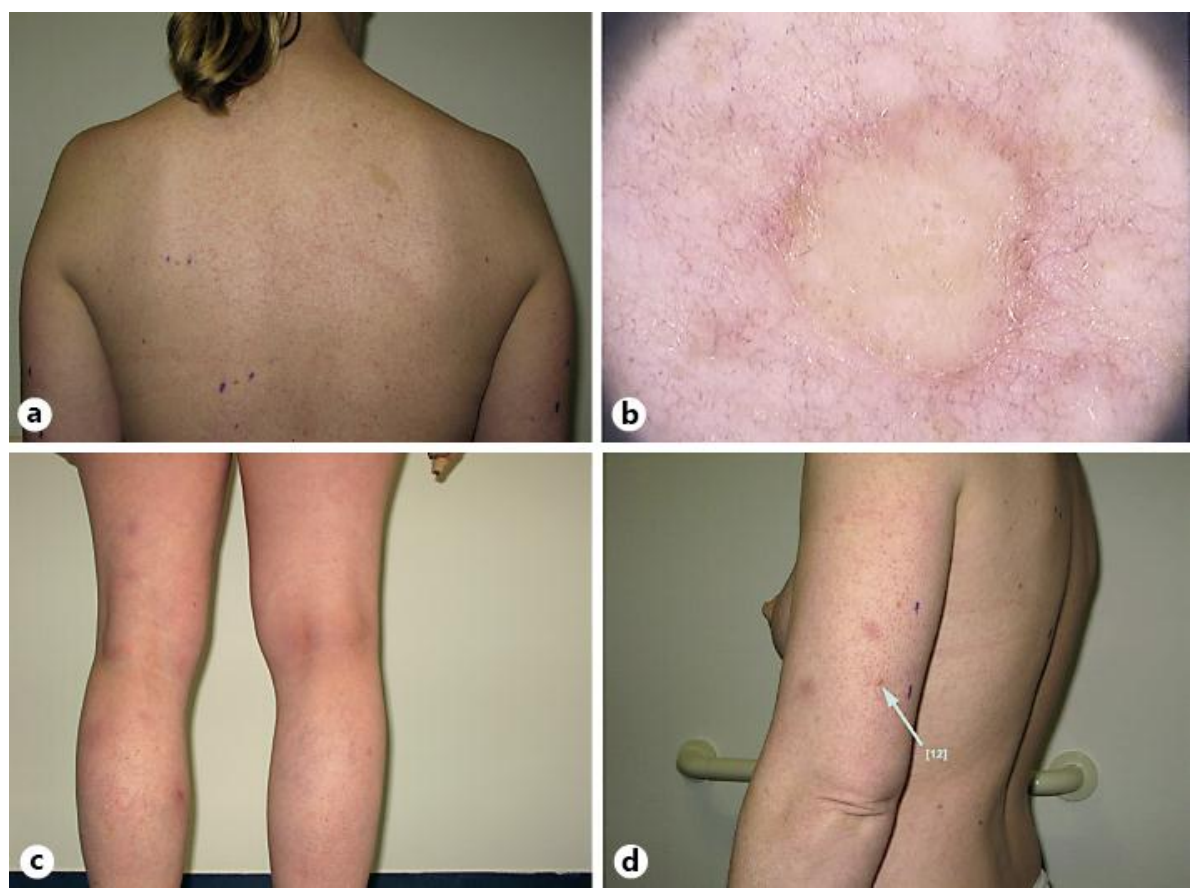

Fig. 1. a Overview: multiple new erythematous papules and plaques on the forearms and neck under therapy with vemurafenib with wart-like appearance. b Dermatoscopic view of an eruptive nevus on the forearm. c Painful erythema nodosum-like lesions and follicular rash of the legs. $\mathbf{d}$ Melanoma in situ on the left forearm.
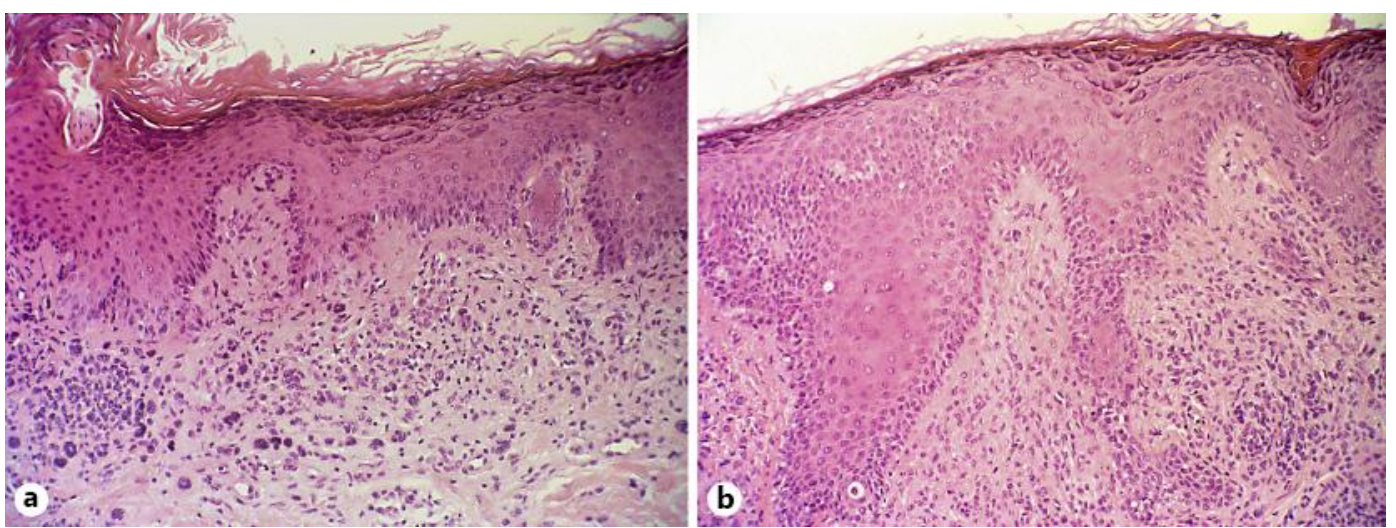

Fig. 2. a Histology after excision of a papulomatous skin lesion with an eruptive nevus in the dermis (HE, 100x). b Histology of the melanoma in situ of the left forearm. 